\title{
Teams Make the Dream Work
}

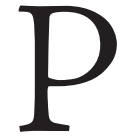

UBLISHING A JOURNAL IS A COMMUNITY EFFORT - ALBEIT MORE A MODERN

quilting bee than a barn-raising, since it requires continued contributions from an evolving group of committed experts. National Volunteer Week fell during the time when this issue of the journal was being prepared, and I am pleased to take this opportunity to celebrate and thank those who made it possible to publish Healthcare Policy/Politiques de Santé over the past year.

We would have nothing to publish without the careful research and thoughtful commentary submitted for consideration by authors from around the globe. This issue provides an excellent example of the range of policy-relevant topics addressed in the journal's pages. Morris Barer, Bob Evans and Lindsay Hedden discuss the evolving approach to entry-topractice of international medical graduates; Ainslie Hildebrand and colleagues highlight the disconnect between therapeutic notes and actual prescribing practice for seniors; and several authors look at the effectiveness of primary care reform. Other papers illuminate the perspectives of patients and those who care for them. For instance, Daniel Harrington, Kathi Wilson and Mark Rosenberg ask about the impact that waiting for a specialist consultation has on patients' lives. Likewise, Ashlinder Gill and colleagues used semistructured interviews with patients, their informal caregivers and family physicians to describe key themes in managing multiple conditions so as to help identify strategies to address care challenges. Then there are explorations of the knowledge creation and exchange process itself, such as the range of research related to appropriateness of care and the use of deliberative dialogues for knowledge exchange.

To ensure effective peer review for such a broad range of topics, we reach out to experts around the world. Last year, 121 reviewers carved time out of their busy schedules to provide feedback on papers considered for publication in this journal. (Please see the list of reviewers on pages 104.) Their feedback was carefully considered by our team of dedicated editors (listed in the journal's masthead on page 6). Our regular editorial team was joined this year by Jean-Louis Denis, who is serving as the guest editor of a special issue on approaches to accountability in the health sector.

These dedicated volunteers - along with Ania Bogacka and her colleagues and freelancers at Longwoods Publishing - make it possible to publish high-quality content in each issue of Healthcare Policy/Politiques de Santé. 
On behalf of our readers, and the many who will ultimately benefit from their thoughtful reading and use of the journal's content, I would like to thank all those who contributed to the success of the journal over the past year. If you are interested in joining us as a reviewer for future issues, please contact us at www.longwoods.com/reviewer-registration/healthcare-policy.

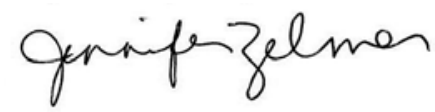

JENNIFER ZELMER, BSC, MA, PHD

Editor-in-chief

\section{Les équipes rendent possible le rêve}

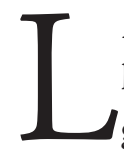
A PUblication D'UNE REVUE EST UN EFFORT COLLECTIF - Plutôt DU RESSORT DE la ruche ouvrière que du chantier de construction -, puisquelle demande l'apport d'un groupe d'experts en constante évolution. La Semaine de l'action bénévole a coïncidé avec la préparation du présent numéro et je tiens à profiter de cette occasion pour remercie tous ceux et celles qui ont rendu possible, au cours de l'année écoulée, la publication de Politiques de Santé/Healthcare Policy.

Il serait impossible de publier cette revue sans les travaux de recherche et les commentaires judicieux soumis par des auteurs provenant d'un peu partout sur la planète. Ce numéro constitue un excellent exemple de l'éventail des sujets abordés par la revue. Morris Barer, Bob Evans et Lindsay Hedden traitent de l'entrée en pratique des étudiants en médecine diplômés à l'étranger; Ainslie Hildebrand et ses collaborateurs font état du décalage entre les notes thérapeutiques et les pratiques de prescription pour les aînés; et plusieurs auteurs se penchent sur l'efficacité des réformes des soins de santé primaires. D'autres articles présentent les points de vue des patients et de leurs soignants. Par exemple, Daniel Harrington, Kathi Wilson et Mark Rosenberg s'intéressent à l'impact de l'attente pour une consultation auprès d'un spécialiste sur la vie des patients. De leur côté, Ashlinder Gill et ses collaborateurs ont mené des entrevues semi-dirigées auprès de patients, de leurs aidants naturels et de leurs médecins de famille pour décrire la gestion des situations de multimorbidité, et ce, afin de définir des stratégies pour 
faire face aux défis en matière de soins. Il y a aussi des études sur les processus de production et d'échange de connaissances, tels que la portée des recherches sur la pertinence des soins ou l'emploi du dialogue délibératif comme stratégie d'échange de connaissances.

Afin d'assurer l'examen par les pairs d'une telle variété de sujets, nous faisons appel à des experts du monde entier. L'année dernière, 121 examinateurs ont donné de leur temps pour fournir des commentaires sur les articles soumis à la publication. (Veuillez consulter la liste des examinateurs à la page 104.) Ces commentaires ont été pris en compte par l'équipe de rédaction (présentée dans le bloc générique, à la page 6). Cette année, Jean-Louis Denis sest aussi joint à léquipe de rédaction à titre de directeur scientifique invité pour un numéro spécial sur les démarches ayant trait à l'obligation de rendre compte dans le secteur de la santé.

Ces bénévoles dévoués - de pair avec Ania Bogacka, ses collègues et des travailleurs pigistes - rendent possible la publication d'un contenu de qualité dans chacun des numéros de Politiques de Santé/Healthcare Policy.

Au nom de nos lecteurs et de tous ceux qui tirent profit de la lecture et du contenu des articles, je voudrais aussi remercier ceux et celles qui ont participé au succès de cette revue au cours de l'année écoulée. Si vous désirez vous joindre à nous comme examinateur pour les prochains numéros, veuillez communiquer avec nous à l'adresse suivante : www.longwoods.com/reviewer-registration/healthcare-policy.

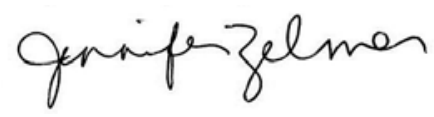

JENNIFER ZELMER, BSC, MA, PHD

Rédactrice en chef 